\title{
Fabrication of a Shear Stress Sensor Matrix Using Standard Printed Circuit Board and Overmoulding Technologies
}

\author{
Ann Monté ${ }^{1}$, Jef Daems ${ }^{1}$, Jeroen Missinne ${ }^{1}$ \\ ${ }^{1}$ Centre for Microsystems Technology (CMST) \\ imec and Ghent University \\ Technologiepark 126, 9052 Gent, Belgium \\ ann.monte@imec.be,jef.daems@ugent.be, jeroen.missinne@ugent.be \\ Corresponding author: Jeroen Missinne
}

\begin{abstract}
In contrast to pressure distributions that can nowadays easily be measured using commercial sensor sheets, this is not yet the case for frictional or shear stresses. Those stresses act parallel to a surface which makes it more challenging to develop suitable sensors. A number of shear sensor prototypes have been reported until now, but realizing a matrix of shear sensors remains a big challenge because of cost and complexity issues. Therefore, this paper presents a fabrication approach for realizing a matrix of shear sensors using standard PCB and moulding technologies. The presented sensor is based on changing the coupling of optical power between a Light Emitting Diode and a set of photodiodes in combination with an overmoulding step, as such realizing a mechanical transducer. To demonstrate the fabrication flow, a first demonstrator incorporating a 5-taxel sensor matrix has been realized, able to record the in-plane shear stress magnitude and direction.
\end{abstract}

Index Terms-frictional force, LED, mirror, optoelectronic, photodiode, sensor matrix, shear stress sensor

\section{INTRODUCTION}

$\mathbf{S}$ everal types of pressure distribution sensors are already commercially available and employed for example for gait analysis. These products consist of a matrix of sensor points which allow detecting the forces or stresses acting perpendicularly to the surface at each point, but are unfortunately not able to detect friction or slippage events. It would therefore be beneficial to add shear stress sensing functionality to those matrices which would result in a full 3D force sensor. Several efforts have been reported to implement shear stress sensors, and some of these are compared by Satu et al. [1], [2]. From this overview, it is clear that shear sensors are much more challenging to realize than pressure sensors, since they need to detect forces acting parallel to a surface. Therefore, many of the concepts that have been reported are rather complex and bulky since they somehow need to allow lateral displacements of sensor elements. Although a single 3D force sensor is already commercially available [3], realizing shear sensors in matrices with a large number of elements requires miniaturization on one hand and scalable manufacturing techniques on the other hand to become economically viable.

Furthermore, recently, there has been a trend to move towards optical sensors, which have the advantage of not being susceptible to electromagnetic interference, of not or only in a limited way being influenced by moisture and which can be designed to be very sensitive or to be very small.

We have previously demonstrated these capabilities by implementing an ultra-thin (about $250 \mu \mathrm{m}$ thick) and very sensitive shear sensor based on the changing coupling between a vertical-cavity surface-emitting laser (VCSEL) and a photodiode [4], which can also be used for multi-axial sensing [5]. Despite the high sensitivity, small footprint, and low thickness of this sensor, implementing it in a matrix configuration is difficult because (i) both the bottom layer and (laterally moving) top layer of the sensor contain interconnections and (ii) because rather costly components (bare die VCSEL and photodiode chips) and fabrication methods (thin-film technologies) were used.

To overcome the first difficulty mentioned above, an implementation based on reflection is desired. Iwasaki et al. have placed a trapezoidal reflective metal frame above a VCSEL centered on top of 4 photodiodes [6]. Cirillo et al. and Wheeler et al. have both used a concept based on 4 emitter-detector pairs covered with a white reflective silicone [7], [8]. Another black silicone is used as encapsulation, to reduce stray light as much as possible. Especially the latter approach using soft silicones instead of metals is promising for applications in contact with the human body for instance.

In parallel, we have been working on a similar approach to make our previously reported shear sensor concept [4] more scalable. Compared to [7], [8], we chose to use 1 LED centered between 4 photodiodes (PDs) per $(\mathrm{x}, \mathrm{y})$-shear sensing pixel, similar to an optical position sensitive detector using 4 photodiode segments. From an optical point of view, this allows sharing PDs between neighboring sensor pixels, eventually requiring only 1 LED and PD per sensor pixel. We also implemented a design without any air between the LED-PD, allowing a sequential build-up approach starting from a standard rigid or flexible printed circuit board (PCB) and ending with an overmoulding step to implement the mechanical layer. Finally, we believe it is important to separate the optical and mechanical design of the sensor since this allows tuning the sensor's sensitivity and dynamic range per 
application by simply selecting a (transparent) deformable layer with a suitable Young's modulus, without the need to redesign the (optical) sensor itself. The optical part of the sensor employs 4 photodiodes for detecting angular deflections of a mirror centered above the LED, while a mechanical layer, in which this mirror is embedded, translates shear forces into mirror rotations.

The current paper provides a detailed overview of the proposed fabrication approach and reports initial sensing results. Future work will focus on implementing a denser matrix with an increased number of sensor elements and on using a harder transducer material to increase the dynamic range.

\section{SENSOR CONCEPT}

\section{A. Optical sensor}

A single taxel configuration is shown in Fig. 1. One LED is flanked with 4 photodiodes (PD) and at a certain height above the LED, a reflective surface (RS) is placed. By implementing an appropriate transducer mechanism (see below), shear stresses will lead to a tilt of the RS and hence the PDs will detect a different amount of optical power, as illustrated in Fig. 2. When normal pressure is acting on the taxel, all PDs will detect the same change in optical power.

The VCSEL light source used in previous designs is replaced by an infrared LED (Vishay VSMY1940ITX01), which is considerably cheaper than a VCSEL. The choice for LEDs instead of VCSELs leads to a larger dynamic range (in tilt angles that can be detected) but lower sensitivity. The designs based on the VCSEL rely on its narrow beamwidth, which causes a large gradient in the light distribution, resulting in a steep response to small changes and hence a high sensitivity but small dynamic range.

An LED in a lens-less surface mount package was chosen which exhibits a Lambertian radiation pattern with a halfintensity beamwidth of $120^{\circ}$ [9]. This implies that the PDs will have to be placed at a sufficient distance from the LED to achieve a good sensitivity and linearity to changes in tilt angle of the RS. The increased distance between the components has the additional advantage that components of neighboring taxels can be shared in order to further reduce the cost of an array implementation. Yet, this requires that the LEDs of neighboring taxels are switched off while measuring the photocurrent so that no optical interference will occur.
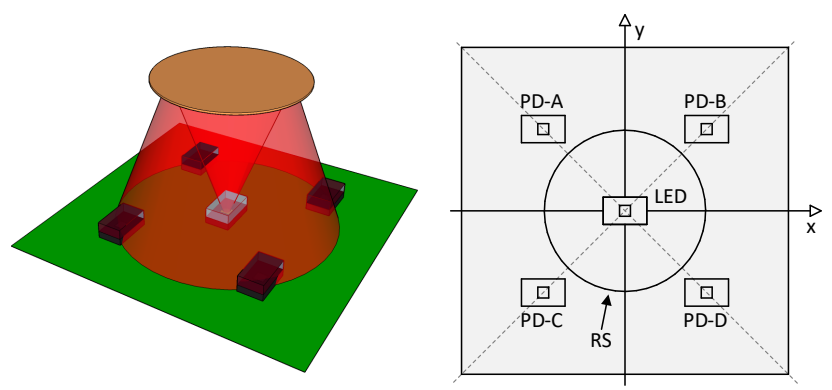

Fig. 1: The configuration of a single taxel.

A theoretical model was used to evaluate the effects of both rotation and translation of the RS as well as to determine the optimal height and diameter of the RS, which are, along with the pitch, the defining dimensions of the taxels structure (see Appendix for details). The pitch is defined as the inter-taxel distance and was chosen to be $10.6 \mathrm{~mm}$ on the demonstrator PCB, corresponding to a LED-PD distance $w=5.30 \mathrm{~mm}$. The $\mathrm{RS}$ diameter used in the demonstrator sensor is $7.4 \mathrm{~mm}$ and the $\mathrm{RS}$ is positioned $7.5 \mathrm{~mm}$ above the PCB, which corresponds to a height of $7.15 \mathrm{~mm}$ above the LED active area.

The theoretical model also allows predicting the sensor response. Fig. 3 shows the relation of the normalized response of the LED-PD pairs along axis B-C as a function of the tilt angle of the RS $(\phi)$. More information can be found in the Appendix.

\section{B. Mechanical transducer layer}

A suitable mechanical transducer structure is required to translate shear stresses into tilt of the RS. To achieve a uniform mechanical response in all horizontal directions, a circularly symmetric design is needed. The most straightforward transducer design consists of a deformable vertical cylinder (or pillar) placed on top of the RS. Shearing forces impinging on the top of the pillar will cause it to topple, thus tilting the RS which is embedded in its base. The transducer structure will be molded using Dow Corning ${ }^{\circledR}$ Sylgard $(184$ PDMS, which is supplied as two-part liquid component kits, allowing it to be poured into a mold after which it cures to a flexible elastomer. For mechanical stability, the base of the pillars is suspended in a continuous block of PDMS. As Sylgard@184 is a relatively incompressible material, the pillars are cut out to a level below the RS in order to reduce the mechanical resistance to RS tilt as shown in Fig. 4. The base material is 5 $\mathrm{mm}$ thick, the bottom part of the pillars is $2.5 \mathrm{~mm}$ high and the pillars extend $9 \mathrm{~mm}$ above the RS.

\section{Readout electronics for multiplexing in a matrix implemen- tation}

As stated in the introduction, the sensor will be implemented in an array configuration. The LEDs will have to switch on and off in turns so that neighboring taxels do not interfere with each other. This requires that the Transimpedance Amplifiers (TIA), which converts the photocurrent to a measurable voltage, has a good step response so that high switching frequencies can be used. Fig. 5 shows the TIA circuit used on the demonstrator board. The PD (Vishay TEMD7100X01) is represented by an equivalent current source in parallel with the combined capacitance of the PD junction and the OPAMP (TL074) input.

It is well known that the stability of a PD-TIA circuit can be improved using a feedback capacitor $\left(C_{f}\right)$. This is desired to achieve maximum stability while retaining a high bandwidth. Therefore, the feedback capacitor has to be dimensioned properly. A rigorous stability analysis led to the following formula for determining $C_{f}$ :

$$
C_{f}=\frac{1}{4 \pi R_{f} f_{G B W P}}\left[1+\sqrt{\left(1+8 \pi R_{f} C_{i} f_{G B W P}\right)}\right]
$$



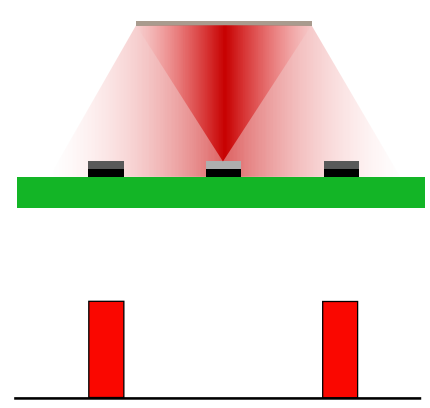

(a)
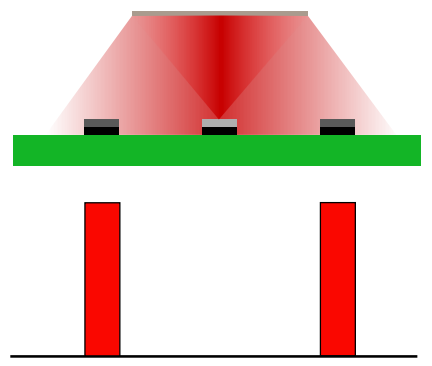

(b)
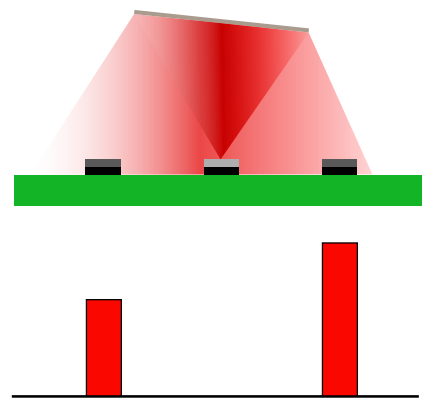

(c)

Fig. 2: Side view of the sensor and visualization of the light intensities per photodiode: (a) default position, (b) downward displacement, (c) tilting of the reflective surface.

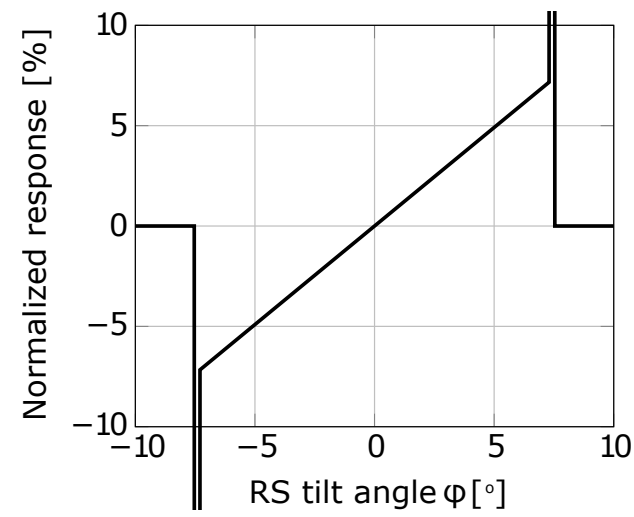

Fig. 3: Normalized sensor response as a function of the RS tilt angle.

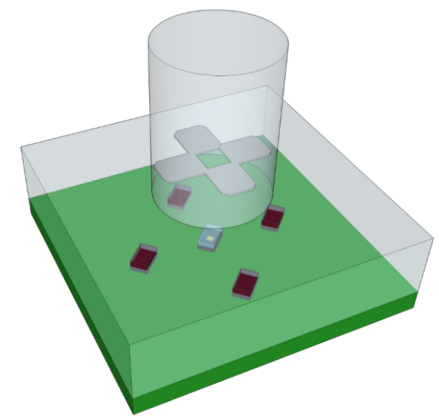

(a) Isometric view

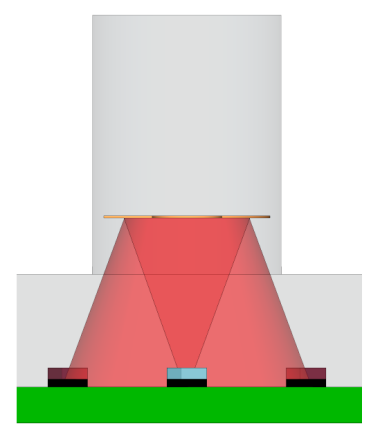

(b) Cross-sectional view
Fig. 4: Transducer structure.

The optimal feedback capacitor for the demonstrator was 1.2 $\mathrm{pF}\left(R_{f}=390 \mathrm{k} \Omega, C_{i}=4.7 \mathrm{pF}, f_{G B P W(\min )}=1.8 \mathrm{MHz}\right)$.

The current demonstrator sensor consists of 5 taxels, yet, this can easily be scaled up. For a high number of taxels, one extra taxel requires one extra LED and one extra PD.

\section{SENSOR FABRICATION}

The proposed sensor is realized by moulding the mechanical transducer layer with RS on top of a (rigid or flexible) printed circuit board on which the sensor LEDs and PDs, and the required driving electronics have been assembled. Therefore,

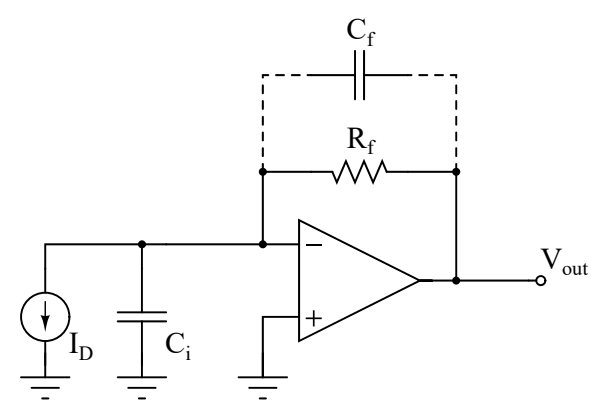

Fig. 5: Stabilized TIA circuit.

only 1 additional overmoulding step is required to realize the sensor starting from a standard printed circuit board. This process is described in detail below.

The demonstrator sensor was implemented on a double sided printed circuit board (PCB). On the top side, the LEDs and PDs were mounted and on the back side all electronics were placed. After assembling all (opto)electronic components using reflow soldering, the PDMS transducer (including the embedded RS) was applied on the top side of the PCB using over-molding. The mold elements were milled out of 10 mm thick Polymethyl methacrylate (PMMA) according to the specifications mentioned above. In order to allow positioning of the RS, the mold is divided into two parts and metal rods are used to align both parts with respect to the PCB. An overview of the transducer layer fabrication flow is shown in Fig. 6. The bottom part of the mold is placed first and contains the part of the pillars that resides on top of the RS, as shown in Fig. 7a and Fig. 6a. Then, the foil with the reflective surfaces is placed on top (Fig. 7b and Fig. 6b). For ease of alignment, the individual RS elements are suspended in a continuous mirror foil (optimont $\mathrm{R}$ S2D high-gloss silver-coated $125 \mu \mathrm{m}$ thick PET foil from Bleher Folientechnik) which is patterned using laser ablation (ND-YAG laser, $\lambda=355 \mathrm{~nm}$, spotsize $=$ $20 \mu \mathrm{m})$. The second mold piece contains the bottom part of the pillars and the base layer and is placed on top of the mirror foil as shown in Fig 7c and Fig. 6b. At this moment, the mold is filled with the PDMS mixture (Fig. 6c) and afterwards it is sealed using the PCB as lid (Fig. 7d and Fig. 6d). In 


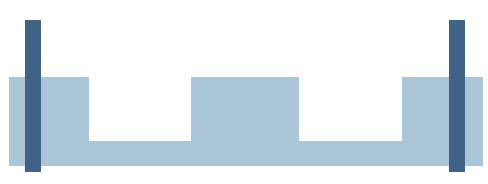

(a)

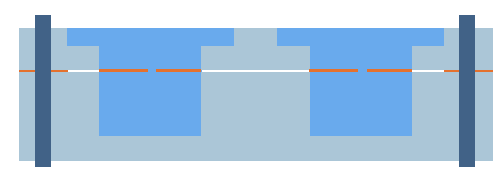

(c)

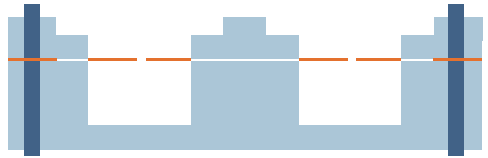

(b)

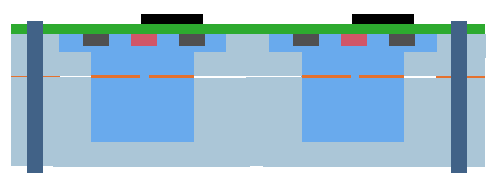

(d)

Fig. 6: Schematic fabrication flow for realizing the PDMS transducer layer on top of the PCB with assembled LEDs and PDs.

order to promote adhesion to the PCB and the RS foil, both were rigorously cleaned using isopropyl alcohol and acetone respectively, and treated with Dow Corning $\AA 1200$ OS primer. Bolts and nuts were used to tightly fix the assembly after which the PDMS was cured at room temperature. After hardening the PDMS, the mold parts were separated from the PCB yielding molded PDMS pillars on the PCB surface as shown in Fig. 7e. To reduce parasitic reflections at the PDMS-air interface, the PDMS surface was covered with an IR-absorbing foil (BKF12, Thorlabs) with openings cut out at the pillar locations (not shown in Fig. 7e).

For a larger volume production, the mold can be clamped on top of the PCB, the liquid PDMS mixture can be injected in the mold and subsequently thermally cured within a few minutes.

\section{SEnSOR Characterization}

\section{A. Methods}

The fabrication process was evaluated by characterizing the resulting shear sensor elements. Therefore, known displacements were applied at the sensor pillars using a Newport PM500-XYZ 3-axis motorized linear stage. A square metal plate attached to a metal control arm was used to contact the sensor taxels from above as illustrated in Fig. 8.

The measurement procedure consisted of moving the contact plate down until it touched the pillars of the 5 taxels and consecutively moving in the XY-plane to induce lateral displacements and tilting of the pillars. To ensure enough grip on the pillars, the contact surface is moved down beyond the contact point by $500 \mu \mathrm{m}$.

First, the sensor response to RS tilt angle was measured in order to compare it with the simulated values. Since the height of the pillars is $11.5 \mathrm{~mm}$, the tilt angle can be approximated by:

$$
\phi=\sin ^{-1} \frac{d}{11.5 m m}
$$

with $d$ the applied lateral displacement in $\mathrm{mm}$ and $\phi$ the resulting estimated RS tilt angle. Note that the actual tilt angle may be slightly lower due to bending of the pillars.
Then, the two-dimensional sensor response was recorded by moving the contact surface along different paths in the XYplane.

The photodiode current was sampled at 2 Msps by the onboard ADC and the obtained ADC values were transferred over USB, after which this raw data was averaged over 100 points on a pc. Each individual taxel was calibrated by dividing the real-time ADC values by the ADC value recorded when no forces were applied on the taxel. This cancels out possible offset differences between individual taxels, caused by placement errors of the PDs and LEDs, slight variations in LED output power, aging effects and differences in stray reflections. Furthermore, the difference between the photocurrents of each photodiode pair is normalized by the total current corresponding to that pair to obtain the relative sensor response. As explained above, the 5 LEDs corresponding with the 5 taxels were turned on and off sequentially, and the photocurrents of the corresponding photodiodes were sampled $100 \mu$ s after turning on the LED. Furthermore, a delay of $10 \mu \mathrm{s}$ was introduced after switching an analog multiplexer allowing the signals to stabilize.

\section{B. Results and Discussion}

1) Recorded signal as a function of mirror tilt: Fig. 9 depicts the recorded signals when a translation along the diagonal axis (i.e. $\mathrm{x}=\mathrm{y}$ or the $\mathrm{BC}$-axis) is applied, using the naming conventions of Fig. 1. This and following graphs show the actually applied displacement on the $\mathrm{x}$-axis and the estimated tilt angle (based on Equation 2) on the secondary $\mathrm{x}$-axis. The sensor behaves as expected, showing an increase in the (B-C) curve when the RS is tilted towards PD-B and a decrease when tilting towards PD-C. Furthermore, the amount of optical power detected by PD-A and PD-D remains virtually the same. Note that saturation of the signal is seen for tilt angles larger than about 7 degrees, corresponding with the moment at which the LED light starts to fall outside the RS boundaries. Since the actual PDs are not point detectors, and because of some stray light, no steep drop in signal is seen as compared to the simulated graph shown in Fig. 3.

It should be noted that the produced demonstrator does not use ideal optical materials and hence does not fully satisfy 


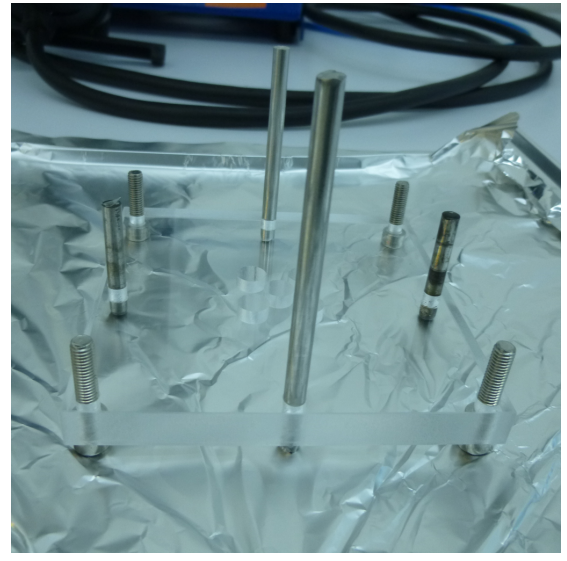

(a)

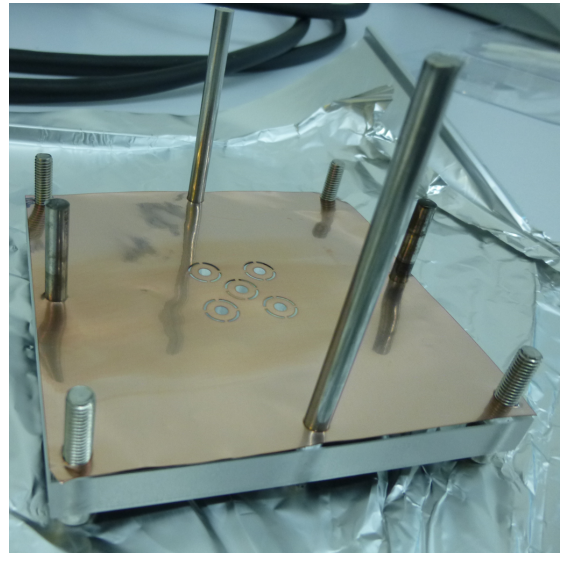

(b)

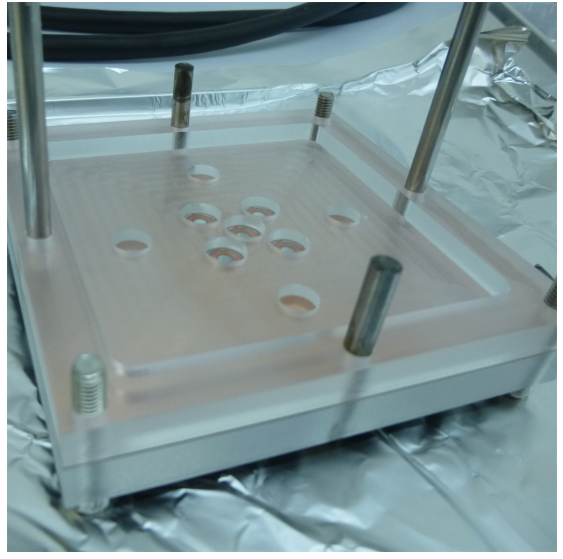

(c)
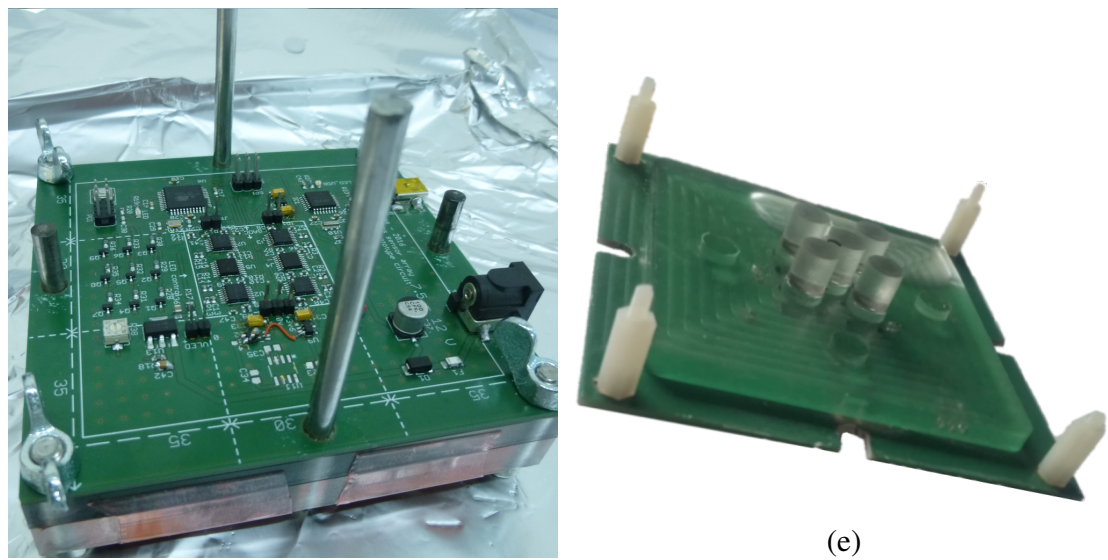

(e)

(d)

Fig. 7: Assembly of the mold for the fabrication of the PDMS transducer layer on top of the PCB with assembled LEDs and PDs.

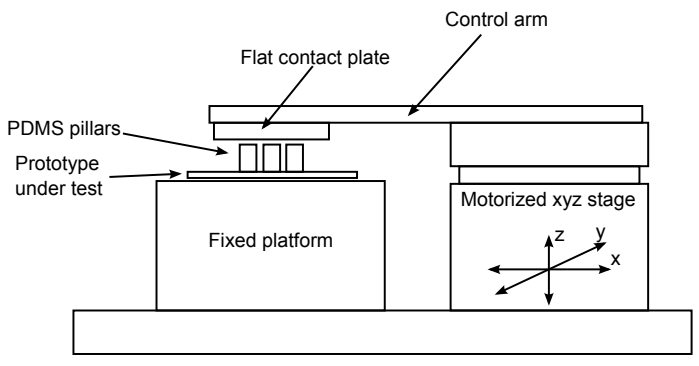

Fig. 8: Side view of the setup used for characterizing the sensor showing the control arm with plate to contact the PDMS pillars and move in the $(\mathrm{x}, \mathrm{y}, \mathrm{z})$-directions.

the assumptions that were made while forming the theoretical model. The reflection on the reflective surface is not purely specular but slightly spread, and the PCB, which should ideally be an absorber of infrared light, reflects a significant amount of light. Furthermore, the PDMS-air interfaces will lead to parasitic reflections. As a result, stray reflections occur which affect every photodiode differently. This issue was largely tackled by using a high-gloss reflective surface, by

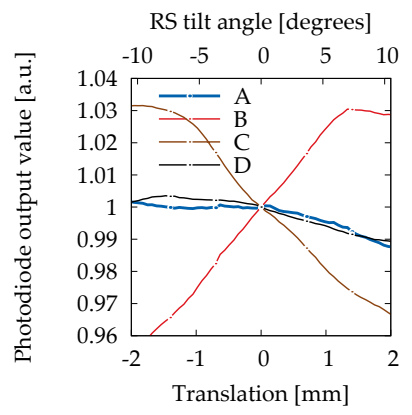

(a) Individual PD values.

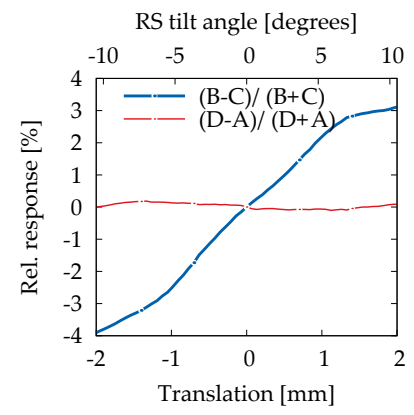

(b) Relative response curves.

Fig. 9: Recorded individual PD values and relative response for tilt along the $(\mathrm{x}=\mathrm{y})$ axis.

the calibration step and by adding the IR-absorbing foil on top of the PDMS. However, since the desired signal power is superimposed on the power of the stray reflections, the relative sensitivity of the sensor drops. While a sensitivity of about $1 \%$ per degree of tilt is expected from the simulation, only $0.4 \%$ per degree was recorded on average.

2) Two-dimensional sensor response: By translating over the x-axis, which bisects the PD axes, both PD pairs 
should generate the same response. This allows evaluating the difference in sensitivity for both axes. Fig. 9 shows the normalized individual PD values and relative sensor response for taxel 2 for translations along the X-axis. Fig. 11 shows the relative sensor response for all other taxels. The response curves for PD pairs B-C and D-A are fairly equal for this taxel, however slight deviations (especially for taxel 5) could be observed when considering all tested taxels on the demonstrator. This points at a slight difference in the component parameters, component positions, or the stray reflections in the bulk PDMS material. Also here, saturation can be seen when going beyond the intended operation range.

Finally, it should be noted that, while the sensor response to tilt angle is defined by the optical design, the actual sensitivity (and linked with that the dynamic range) with respect to shear stresses is dependent on the choice of the transducer material. In this paper, a rather low modulus type of in-house available PDMS was used yielding a very sensitive sensor, but with a limited dynamic range. The relation between applied displacement and resulting shear force was determined by pushing a single PDMS pillar using a probe on which a force sensor was mounted. From this experiment, a sensitivity of $5 \% / \mathrm{N}$ and dynamic range of $\pm 360 \mathrm{mN}$ was obtained. For many applications, this range would be too limited but it can be increased by using higher modulus transducer materials and by reducing the pillar height.

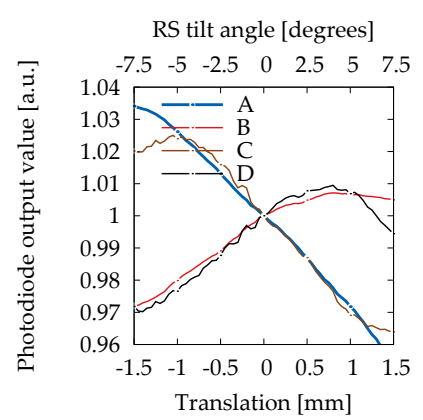

(a) Individual PD values.

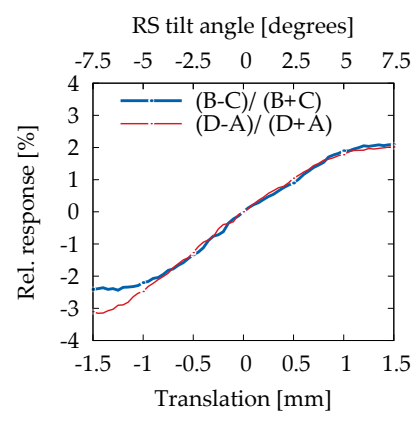

(b) Relative response curves.
Fig. 10: Recorded individual PD values and relative response for translation along the $\mathrm{x}$-axis (taxel 2).

\section{CONCLUSION}

We have shown that it is possible to realize an optical shear stress sensor matrix using standard PCB and moulding technologies. Each sensor point consists of an LED and 4 surrounding photodiodes, assembled on a PCB, which are covered by a reflective surface embedded in a deformable PDMS pillar transducer which is moulded on top of the PCB. A lateral displacement of the pillar top surface leads to tilting of the reflective surface and as such a changing reflected power detected on each photodiode, enabling detection of both shear magnitude and direction. The sensor response to lateral displacements was determined for the current demonstrator and it depends heavily on the mechanical properties of the pillar material. In the current paper, a rather soft in-house

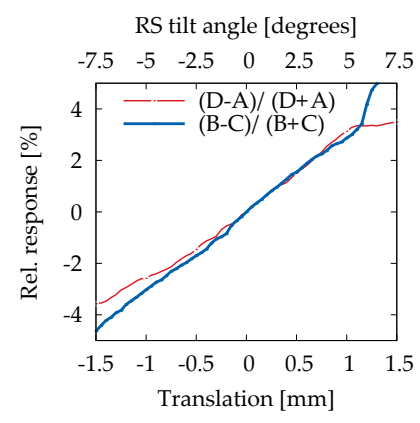

(a) Taxel 1 .

RS tilt angle [degrees]

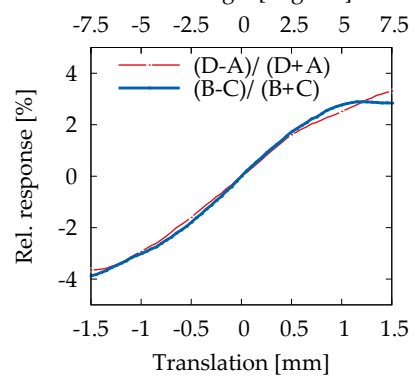

(c) Taxel 4.

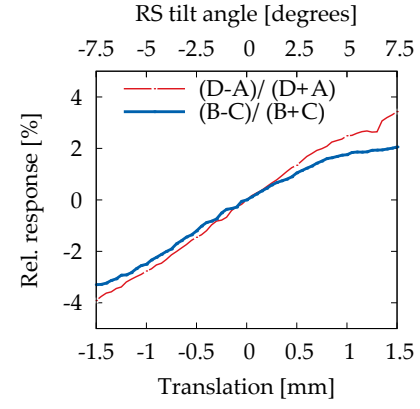

(b) Taxel 3 .

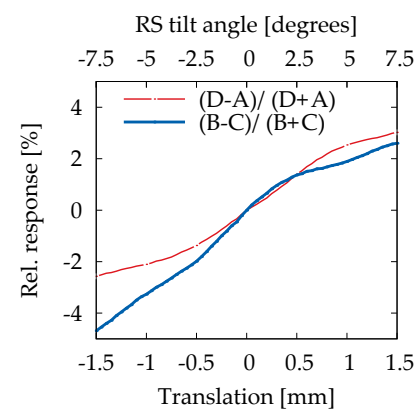

(d) Taxel 5 .
Fig. 11: Recorded relative response for translation along the $\mathrm{x}$-axis.

available PDMS (Sylgard@184) was used to demonstrate the fabrication flow, and this resulted in a high sensitivity to shear stress $(5 \% / \mathrm{N})$, but correspondingly limited sensor range $( \pm 360 \mathrm{mN})$. In future realizations, higher Young's modulus transducer materials will be investigated to extend the dynamic range required for the typical applications. Furthermore, the sensor elements can be miniaturized (especially the pillar with reflective surface) to allow implementing a denser matrix with an increased number of sensor elements.

\section{APPENDIX}

\section{D OPTICAL SENSOR MODEL}

In order to evaluate the proposed sensor structure, a theoretical model was devised allowing to simulate the variation in PD currents. As the light reflection is invariant with respect to RS translations along directions tangent to the RS itself (for translations small enough compared to the dimensions of the $\mathrm{RS}$ ) and rotation around the RS normal axis, it is clear that the device shown in Fig. 1 is only sensitive to translations normal to the RS and rotations around RS tangent axes (if the RS is sufficiently large). Hence three parameters describe the actual RS configuration (1 translation and two rotations), which can be estimated by using a minimum number of 3 PDs [10]. In the current implementation, 4 PDs are used which simplifies the derivation of the RS orientation due to the obtained symmetry, and leads to a push-pull configuration, reducing the effects acting on all 4 PDs at the same time (such as temperature and common-mode noise). Fig. 2 shows a cross section of the optical structure and the expected results of RS reorientation. Vertical movement will affect both photocurrents in the same way, while tilt will create differences in both responses. It is 


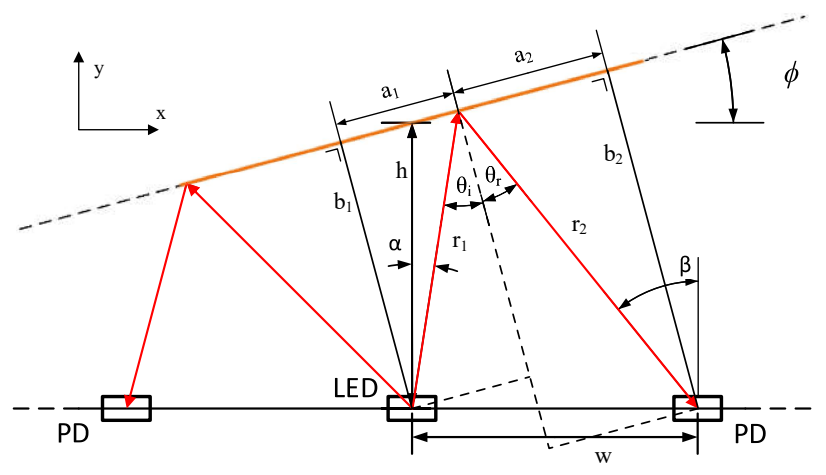

Fig. 12: 2D geometrical representation of the optical structure.

important to understand that the optical path length will be the dominating factor so that the photocurrents rise when the RS comes closer vertically.

As long as the tilt angle of the RS is small, the two rotations defining the RS orientation will have virtually no influence on each other. As a result, the optical properties of the sensor can be analyzed using a 2D model. Fig. 12 shows a 2D geometrical representation of the optical structure. The figure shows how the light propagates from the LED to the PDs. For the derivation of the model, it is assumed that all surfaces other than the RS perfectly absorb the light and that the reflection on the RS is specular. That way, only the first reflection has to be taken into account.

It is possible to describe the propagation by means of a coupling coefficient $\kappa$ that expresses the relation between the transmitted and the received light intensity. The coupling coefficient depends on four factors:

1) $\alpha$ - The radiation pattern of the LED. The LED is modeled as a point source with a Lambertian radiation pattern:

$$
I=I_{0} \cos (\alpha)
$$

with $I_{0}$ the radiant intensity normal to the source.

2) $r$ - The total length of the optical path between the LED and a PD. In this case, the light intensity reduces with increasing $r$ due to spherical expansion, which can be modeled by a path loss factor $4 \pi / r^{2}$.

3) $\theta_{i}, \theta_{r}$ - The incidence angle and the emission angle of the reflected light measured from the normal of the RS, which determine the complex reflection coefficient $\Gamma$ of the RS. $\Gamma$ is calculated using the Fresnell equations. Note that the law of reflection states that $\theta_{i}=\theta_{r}$.

4) $\beta$ - The incidence angle of the light on the photodiode. Because of the physical similarity between PIN photodiodes and LEDs, and the absence of focusing lenses, the photodiodes will have an angular response that can also be modeled by a cosine function $\cos (\beta)$. Another way to understand this is that the projected photodiode area depends on $\beta$ in the same way.
Combining these four terms allows to write down a formula for the coupling coefficient:

$$
\kappa=\frac{4 \pi}{r^{2}} \cos (\alpha) \cos (\beta) \Re\left\{\Gamma\left(\theta_{i}\right)\right\}
$$

Note that this equation does not account for the relation between the LED current and the intensity of the emitted light or the resulting photocurrent. These relationships can be taken into account as constant factors which can be derived from the datasheets of the selected components. An exact result for $\kappa$ can only be obtained by integrating this equation over the active area of the PD, but as long as the active area is small compared to the other dimensions of the taxel, $\kappa$ can be approximated by assuming rays incident on the center of the active area and dropping the integration.

It then only remains to derive these parameters from the drawing in Fig. 12. To tackle this problem, it is convenient to imagine the rectangles with dimensions $\left(a_{1}, b_{1}\right)$ and $\left(a_{2}, b_{2}\right)$, completed in the drawing by the dotted lines. The law of reflection states that:

$$
\theta_{i}=\theta_{r}
$$

This requires that the rectangles are scaled versions of each other, which requires that:

$$
\frac{b_{1}}{b_{2}}=\frac{a_{1}}{a_{2}}
$$

The values of $b_{1}, b_{2}$ and the sum $\left(a_{1}+a_{2}\right)$ can then be found:

$$
\begin{gathered}
a_{1}+a_{2}=w \cos (\phi) \\
b_{1}=h \cos (\phi) \\
b_{2}=h \cos (\phi)+w \sin (\phi)
\end{gathered}
$$

where $w$ is the distance between the LED and a PD and $h$ is the height of the center of the RS with respect to the active areas of the components. By combining equations (6) to (9), the following equations for $a_{1}$ and $a_{2}$ can be found:

$$
\begin{gathered}
a_{1}=\frac{w h \cos ^{2}(\phi)}{2 h \cos (\phi)+w \sin (\phi)} \\
a_{2}=\frac{w \cos (\phi)(h \cos (\phi)+w \sin (\phi))}{2 h \cos (\phi)+w \sin (\phi)}
\end{gathered}
$$

Knowing the dimensions of the rectangles, the required parameters to determine $\kappa$ can be derived as follows:

$$
\begin{gathered}
r=r_{1}+r_{2}=\sqrt{\left(a_{1}\right)^{2}+\left(b_{1}\right)^{2}}+\sqrt{\left(a_{2}\right)^{2}+\left(b_{2}\right)^{2}} \\
\theta_{i}=\theta_{r}=\frac{\pi}{2}-\arctan \left(\frac{b_{1}}{a_{1}}\right)=\frac{\pi}{2}-\arctan \left(\frac{b_{2}}{a_{2}}\right) \\
\theta_{L E D}=\theta_{i}-\phi \\
\theta_{P D}=2 \theta_{i}-\theta_{L E D}
\end{gathered}
$$

Substituting these values into Equation (4) returns the coupling coefficient for the rightmost photodiode in Fig. 12. Due to the symmetry of the structure, the result for the leftmost photodiode can be obtained using the same equations by changing the sign of $\phi$. 
The theoretical model was used to evaluate the effects of both rotation and translation of the RS as well as to determine the optimal height and diameter of the RS, which are, along with the pitch, the defining dimensions of the taxels structure. The pitch is defined as the inter-taxel distance and is 10.6 $\mathrm{mm}$ on the demonstrator PCB, corresponding to a LEDPD distance $w=5.30 \mathrm{~mm}$. The RS diameter used in the demonstrator sensor is $7.4 \mathrm{~mm}$ and the RS is positioned 7.5 $\mathrm{mm}$ above the PCB, which corresponds to a height of 7.15 $\mathrm{mm}$ above the LED active area.

The resulting coupling coefficients $\kappa$ as a function of RS tilt angle are shown in Fig. 13. The point of reflection moves along the RS surface as a function of the RS tilt angle. When this point falls outside of the RS boundaries, no reflected light can reach the PD and $\kappa$ drops to 0 . In reality, this transition is much smoother as the LED and PD active areas are not actually points but small rectangular surfaces.

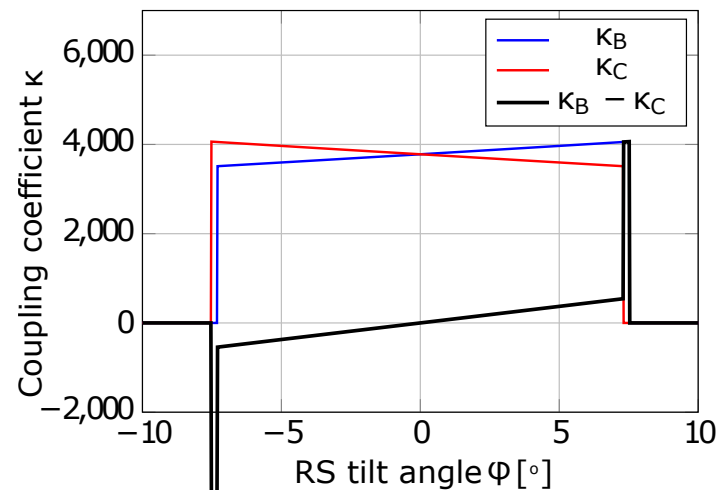

Fig. 13: Coupling coefficients for both photodiodes along with the difference $\kappa_{B}-\kappa_{C}$ as a function of the RS tilt angle.

As stated earlier, the sensor concept mainly relies on the variation in optical path length, which is in general a nonlinear term. Within the specified operation range, the nonlinearity of the path loss term is compensated by the cosine terms, resulting in an almost linear relation with the tilt angle. The tilt angle can thus be extracted from the coupling coefficients in a straightforward way. The tilt angle along the RS tangent axes parallel to PD pair (CB) is denoted $\phi_{C B}$ and is determined by the PD values of PD-A and PD-D. Similar conventions hold for the other axis, resulting in the following equations used to derive the tilt angles:

$$
\begin{gathered}
\phi_{C B}=A \frac{\kappa_{A}-\kappa_{D}}{\kappa_{A}+\kappa_{D}} \\
\phi_{A D}=A \frac{\kappa_{B}-\kappa_{C}}{\kappa_{B}+\kappa_{C}}
\end{gathered}
$$

where $A$ is a calibration constant taking into account the efficiency of the LED and responsivity of the PD. From these equations, the normalized response graph as a function of RS tilt angle can be obtained (i.e. Equation 16 or 17 with $A=1$ ), which is plotted in Fig. 3. It can therefore be seen that, under ideal circumstances, the relative response is about $1 \%$ per degree tilt.

\section{ACKNOWLEDGMENT}

The authors thank the Hercules foundation to provide funding for the APPLIE4MOS polymer prototyping line used in this research.

\section{REFERENCES}

[1] R. Satu and L. Jukka, "Plantar shear stress measurements A review," Clinical Biomechanics, pp. 475-483, 2014.

[2] Z. Ting, L. Hong, J. Li, F. Shaowei, and Y. Jing, "Development of a Flexible 3-D Tactile Sensor System for Anthropomorphic Artificial Hand," Sensors Journal, IEEE, vol. 13, no. 2, pp. 510-518, 2013.

[3] OptoForce. (2018, Mar.) Robot sensor - sensor of touch to industrial robots. [Online]. Available: https://optoforce.com/

[4] J. Missinne, E. Bosman, B. Van Hoe, G. Van Steenberge, S. Kalathimekkad, P. Van Daele, and J. Vanfleteren, "Flexible shear sensor based on embedded optoelectronic components," IEEE Photonics Technology Letters, vol. 23, no. 12, pp. 771-773, 2011.

[5] J. Missinne, P. Kostov, B. Van Hoe, E. Bosman, W. Gaberl, H. Zimmermann, and G. Van Steenberge, "Ultra-thin multi-axial shear stress sensor based on a segmented photodiode," 2013 IEEE Photonics Conference, IPC 2013, pp. 606-607, 2013.

[6] T. Iwasaki, T. Takeshita, Y. Arinaga, K. Uemura, H. Ando, S. Takeuchi, M. Furue, E. Higurashi, and R. Sawada, "Shearing force measurement device with a built-in integrated micro displacement sensor," Sensors and Actuators A: Physical, vol. 221, pp. 1-8, 2015.

[7] A. Cirillo, P. Cirillo, G. De Maria, C. Natale, and S. Pirozzi, "An artificial skin based on optoelectronic technology," Sensors and Actuators A: Physical, vol. 212, no. JUNE, pp. 110-122, 2014.

[8] J. Wheeler, A. Mazumdar, L. Marron, K. Dullea, J. Sanders, and K. Allyn, "A pressure and shear sensing liner for prosthetic sockets," pp. 2026-2029, Aug 2016.

[9] Labsphere, "The Radiometry of Light Emitting Diodes," Labsphere, Inc., Tech. Rep., 2008. [Online]. Available: https://www.labsphere.com/wp-content/uploads/2015/02/TheRadiometry-of-Light-Emitting-Diodes-LEDs.pdf

[10] G. Palli, L. Moriello, U. Scarcia, and C. Melchiorri, "Sensors and Actuators A : Physical Development of an optoelectronic 6-axis force / torque sensor for robotic applications," Sensors \& Actuators: A. Physical, vol. 220, pp. 333-346, 2014.

Jeroen Missinne received the Master of Science degree in Electrical Engineering in 2007 and his PhD in Electrical Engineering in 2011, both at Ghent University, Belgium. He is currently employed as part-time professor at Ghent University and employed at the CMST (Center for MicroSystems Technology), an imec-affiliated research lab at Ghent University. His research involves micro-/nanofabrication technologies in general, and in particular polymer optical waveguides, integration of optical and optoelectronic components and optical (bio)sensors.

Ann Monté was born in Zottegem, Belgium, in 1980. She received the M.Sc. and Ph.D. degrees in electronics engineering from the University of Ghent, Belgium, in 2003 and 2008 respectively. Until today she is affiliated with the Inter-University Microelectronics Centre (imec), in the Cmst group at the University of Ghent. She is involved in research on the design of driver electronics for both displays and lighting as well as in the design of integrated sensor systems. She is author or co-author of 25 papers in international technical journals and conference proceedings.

Jef Daems received the Master of Science degree in Electrical Engineering in 2016 at Ghent University, Belgium. He is currently employed as a Hardware Board Design Expert Engineer at Nokia in Antwerp. 